\title{
Diagnosis and management of hypocalcaemia
}

\author{
Mark S Cooper, Neil J L Gittoes
}

Division of Medical Sciences, University of Birmingham,

Birmingham B15 2TH

Correspondence to: N J L Gittoes,

Department of Endocrinology,

Queen Elizabeth Hospital,

Birmingham B15 2TH

n.j.gittoes@bham.ac.uk

BMJ 2008;336:1298-302

doi:10.1136/bmj.39582.589433.BE
Hypocalcaemia is a potentially life threatening biochemical abnormality that carries risks for serious errors in diagnosis and treatment. Hypocalcaemia presents in primary and secondary care; it has a prevalence of $18 \%$ in all patients in hospital and $85 \%$ in the intensive care unit. ${ }^{12}$ The most common cause of hypocalcaemia in primary care is vitamin $\mathrm{D}$ deficiency, which-depending on population demographicsmay have a prevalence as high as 50\%. ${ }^{3}$ Hypocalcaemia may be an asymptomatic laboratory finding or a life threatening metabolic disturbance. Acute hypocalcaemia can result in severe symptoms that require rapid admission to hospital and correction. In contrast, when hypocalcaemia develops slowly patients can be surprisingly free of symptoms. This review will help clinicians to optimise the diagnosis and management of patients with hypocalcaemia. Because hypocalcaemia often presents as an emergency and symptoms are rapidly reversed by giving calcium, the evidence base for managing acute hypocalcaemia is mostly based on experience rather than controlled trials.

\section{Who gets hypocalcaemia and why?}

The concentration of calcium in the serum (normal range $2.10-2.60 \mathrm{mmol} / \mathrm{l})$ is regulated by the action of parathyroid hormone and vitamin D on the kidneys, bones, and gastrointestinal tract (fig 1). Parathyroid hormone stimulates calcium resorption in the kidney and calcium release from bone. It also stimulates renal production of 1,25-dihydroxyvitamin $\mathrm{D}$ (calcitriol) from 25-hydroxyvitamin D. 1,25-Dihydroxyvitamin $\mathrm{D}$ is the most active form of vitamin $\mathrm{D}$, and it acts on the gastrointestinal tract to increase calcium absorption. Vitamin D is obtained mainly through synthesis in the

\section{SOURCES AND SELECTION CRITERIA}

We searched PubMed for articles whose titles included the terms "hypocalcaemia", "hypoparathyroidism", or "osteomalacia" and restricted the search to articles published in English in the previous 10 years. We individually reviewed the titles of the resulting 578 articles to identify major themes. Additional searches were made of contemporary textbooks. skin, with a small contribution from the diet. Skin synthesis requires exposure to ultraviolet light and is reduced by skin pigmentation.

Hypocalcaemia with low vitamin $D$ concentrations Hypocalcaemia can occur in people whose exposure to ultraviolet light is low, especially those with a diet that is poor in vitamin D. Vitamin D requirements increase during and after pregnancy. Low maternal vitamin D concentrations are associated with hypocalcaemia in breastfed children. ${ }^{4}$

Hypocalcaemia caused by vitamin D deficiency is seen in some patients taking antiepileptic drugs, which induce enzymes that increase vitamin D metabolism. ${ }^{5}$ In a study of institutionalised children with poorly controlled epilepsy, the prevalence of vitamin D deficiency was $75 \% .{ }^{6}$ Poor nutrition and minimal exposure to the sun probably contributed to the osteomalacia.

Patients with small intestinal disease- - such as coeliac disease - where dietary calcium and vitamin D absorption are reduced, may also present with hypocalcaemia. Occasionally, hypocalcaemia is seen in people with a profoundly low dietary intake of calcium whose vitamin $\mathrm{D}$ concentrations are within the normal range.

\section{Hypocalcaemia with reduced parathyroid hormone} function

Hypocalcaemia occurs in patients with impaired function of the parathyroid glands. This is most common after thyroid or parathyroid surgery, but it can be idiopathic-mostly in young adults and less often as part of a genetic syndrome, such as autoimmune polyglandular syndrome type $1 .^{7}$ Reduced concentrations of parathyroid hormone lead to excessive renal calcium loss and reduced intestinal absorption of calcium secondary to decreased production of 1,25-dihydroxyvitamin D. Occasionally, tissue resistance to parathyroid hormone can produce a biochemical pattern similar to hypoparathyroidism, even though concentrations of the hormone are high. Such "pseudohypoparathyroidism" is caused by failure of parathyroid hormone to activate its signalling 


\begin{tabular}{|c|c|c|c|c|}
\hline \multicolumn{5}{|l|}{ Causes of hypocalcaemia } \\
\hline \multirow[b]{2}{*}{ Causes } & \multicolumn{4}{|c|}{ Serum concentrations of markers } \\
\hline & Parathyroid hormone & Alkaline phosphatase & Phosphate & 25-Hydroxyvitamin D \\
\hline \multicolumn{5}{|l|}{ Common } \\
\hline Vitamin D deficiency as a result of limited exposure to ultraviolet light or low dietary intake & High & Normal or high & Low & Low \\
\hline Vitamin D deficiency as a result of malabsorption & High & Normal or high & Low & Low \\
\hline Hypoparathyroidism as a result of surgery & Low & Normal & High & Normal \\
\hline Hypoparathyroidism as a result of autoimmune disease & Low & Normal & High & Normal \\
\hline Renal disease & High & Normal or high & High & Normal or low \\
\hline \multicolumn{5}{|l|}{ Rare } \\
\hline Parathyroid hormone resistance & High & Normal & High & Normal \\
\hline Vitamin D resistance & High & Normal & Low & Normal \\
\hline Autosomal dominant hypocalcaemia & Normal & Normal & Normal & Normal \\
\hline Hypomagnesaemia & Normal or low & Normal & Normal & Normal or low \\
\hline Sclerotic metastases & High & High & Low & Normal \\
\hline \multicolumn{5}{|l|}{ Other } \\
\hline \multicolumn{5}{|l|}{ Hungry bone syndrome after parathyroidectomy for hyperparathyroidism } \\
\hline \multicolumn{5}{|l|}{ Infusion of phosphate or calcium chelators, such as citrate, with massive blood transfusion } \\
\hline \multicolumn{5}{|l|}{ Critical illness } \\
\hline $\begin{array}{l}\text { After intravenous treatment with bisphosphonates, especially high dose treatment in } \\
\text { vitamin D deficient patients }\end{array}$ & & & & \\
\hline
\end{tabular}

pathways. ${ }^{8}$ Pseudohypoparathyroidism is a genetically heterogeneous condition, with some patients having skeletal abnormalities (Albright's hereditary osteodystrophy; fig 2), that can occur in other family members independent of any abnormality of serum calcium. The presence of these features and normal calcium biochemistry is termed pseudopseudohypoparathyroidism.

Severe hypocalcaemia has been reported in patients with pre-existing vitamin $\mathrm{D}$ deficiency who receive intravenous bisphosphonates because these compounds

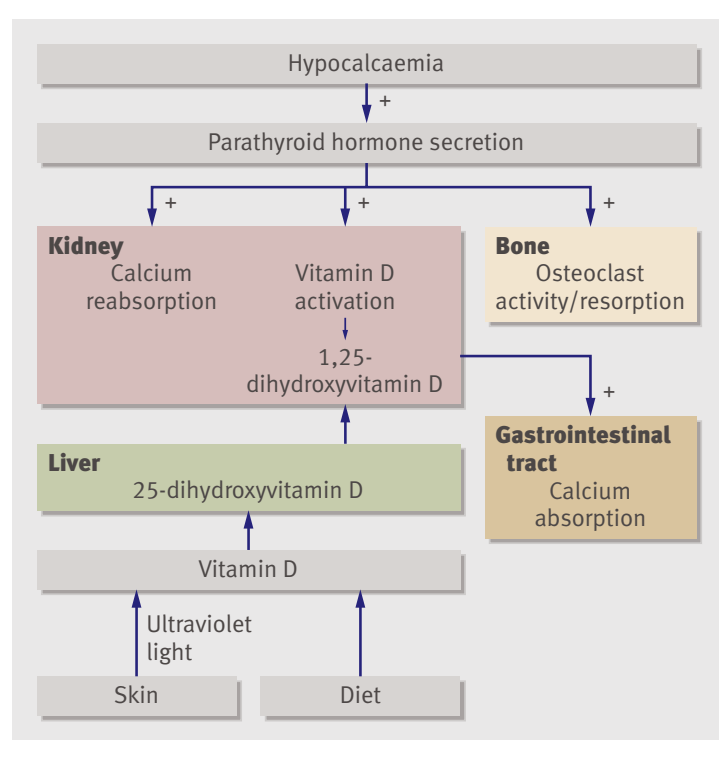

Fig 1 Regulation of serum calcium. Parathyroid hormone and vitamin D normally interact to protect against hypocalcaemia. Problems at any level can lead to low serum calcium, but the most common problems are vitamin D deficiency and hypoparathyroidism block bone resorption..$^{9}$ Table 1 outlines common and less common causes of hypocalcaemia.

\section{How does hypocalcaemia present in clinical practice?}

Extracellular calcium concentrations are important for the normal functioning of muscles and nerves. Thus, classic symptoms of hypocalcaemia are neuromuscular excitability in the form of muscle twitching, spasms, tingling, and numbness. Carpopedal spasm is characteristic but in severe cases can progress to tetany, seizures, and cardiac dysrhythmias. In patients without overt signs, underlying neuromuscular excitability can become evident with provocation-tapping the parotid gland over the facial nerve can induce facial muscle spasm (Chvostek's sign; fig 3). However, 10\% of normal people have a positive Chvostek's sign. Conversely, a small study of patients with hypoparathyroidism and biochemically confirmed hypocalcaemia found that 29\% were negative for Chvostek's sign, which makes this test a poor discriminator. ${ }^{10}$

Mild hypoxia induced by inflation of a blood pressure cuff can precipitate carpopedal spasm (Trousseau's sign; fig 4). Trousseau's sign is relatively specific for hypocalcaemia - $94 \%$ of hypocalcaemic patients display a positive sign, compared with $1 \%$ of normocalcaemic people. ${ }^{10}$

The development of neuromuscular excitability depends on both the absolute concentration of calcium and how rapidly it falls. Rapid falls in calcium-for example, after the removal of a parathyroid adenoma - are often associated with symptoms, whereas patients who develop hypocalcaemia gradually can be surprisingly free of symptoms, and the diagnosis may only become evident as an incidental biochemical finding. Longstanding hypocalcaemia, even without neuromuscular symptoms, is associated with the 

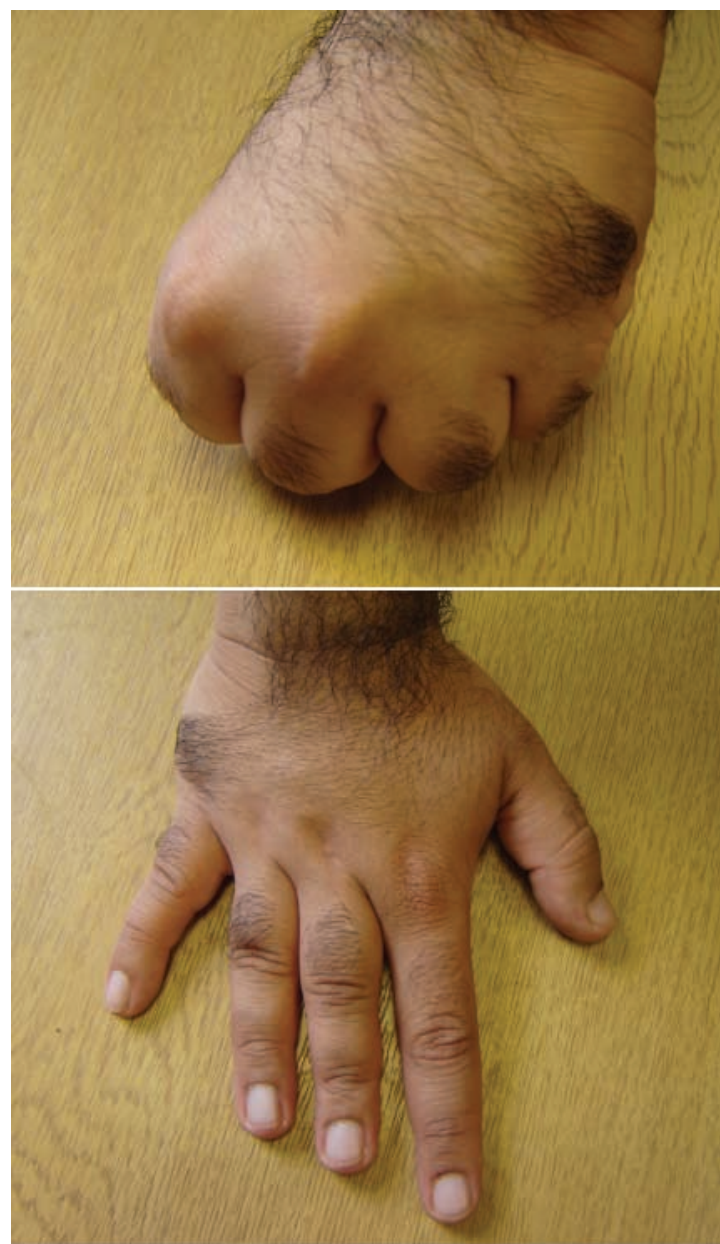

Fig 2 | Albright's hereditary osteodystrophy. Abnormalities of skeletal development are seen in some patients with pseudohypoparathyroidism. The images show shortening of the metacarpal bones that can occur in patients with Albright's syndrome or in patients without abnormalities of calcium or parathyroid hormone (pseudopseudohypoparathyroidism). Images courtesy of Muhammad Ali Karamat

development of neuropsychiatric symptoms,${ }^{13}$ cataract formation, and occasionally raised intracranial pressure. $^{14}$

\section{What is clinically relevant hypocalcaemia?}

The serum calcium concentration must be interpreted in relation to serum albumin. Serum calcium exists in an ionised form $(50 \%)$ or is bound to albumin or other ions. Only ionised calcium is biologically important. Various factors alter the ratio of ionised calcium to bound calcium, but the most important factor is the albumin concentration. Many medical conditions cause a decrease in serum albumin. Serum calcium concentrations are therefore "corrected" to a reference albumin concentration of $40 \mathrm{~g} / \mathrm{l}$, and for every $1 \mathrm{~g} / \mathrm{l}$ of albumin above or below this value, the calcium is adjusted by decreasing or increasing by $0.02 \mathrm{mmol} / \mathrm{l} .{ }^{15}$ For example, a calcium concentration of $2.05 \mathrm{mmol} / \mathrm{l}$ with an albumin concentration of $35 \mathrm{~g} / 1$ would be corrected to $2.15 \mathrm{mmol} / \mathrm{l}$, which

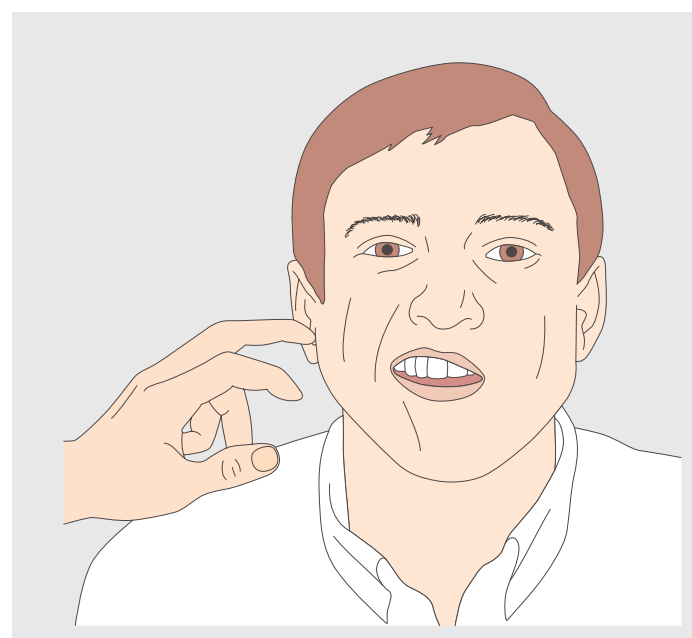

Ask the patient to relax his facial nerves. Next, stand directly in front of him and tap the facial nerve either just anterior to the earlobe or below the zygomatic arch and the corner of the mouth. A positive response varies from twitching of the lip at the corner of the mouth to spasm of all facial muscles, depending on the severity of hypocalcaemia

\section{Fig 3 | Eliciting Chvostek's sign. Adapted from Springhouse ${ }^{11}$}

would correct the hypocalcaemic value to normal. This estimation is often used, but it can be unreliable in some situations, such as critical illness. ${ }^{16}$ If it is doubtful whether total calcium reflects ionised calcium, the ionised value can be checked directly. Other situations in which the assessment of serum calcium may be inappropriately low include recent use of certain gadolinium contrast agents and contamination of blood samples by EDTA.

\section{Which tests help make the diagnosis and define the cause of hypocalcaemia?}

If the cause of hypocalcaemia is not clinically obvious the most important investigation is to measure serum parathyroid hormone. A standard biochemical profile, a parathyroid hormone measurement, and the clinical history will usually provide the likely cause of hypocalcaemia. Figure 5 shows an algorithm for investigating hypocalcaemia.

In true hypocalcaemia, parathyroid hormone concentrations should be high if the parathyroids are appropriately responding to the reduced negative

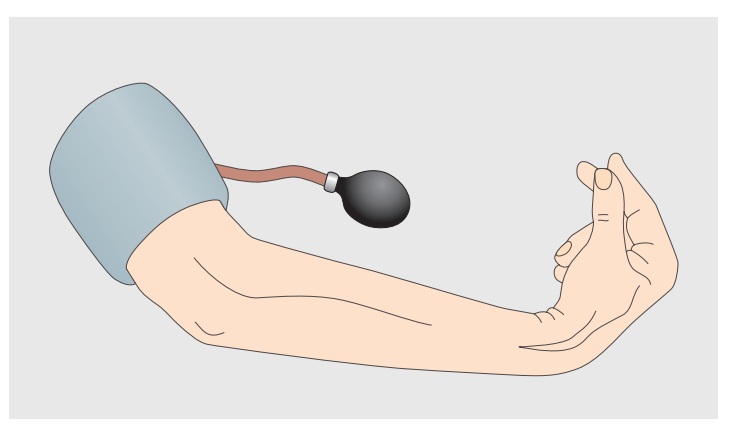

Fig 4 | Eliciting Trousseau's sign. Adapted from Netter $^{12}$ 


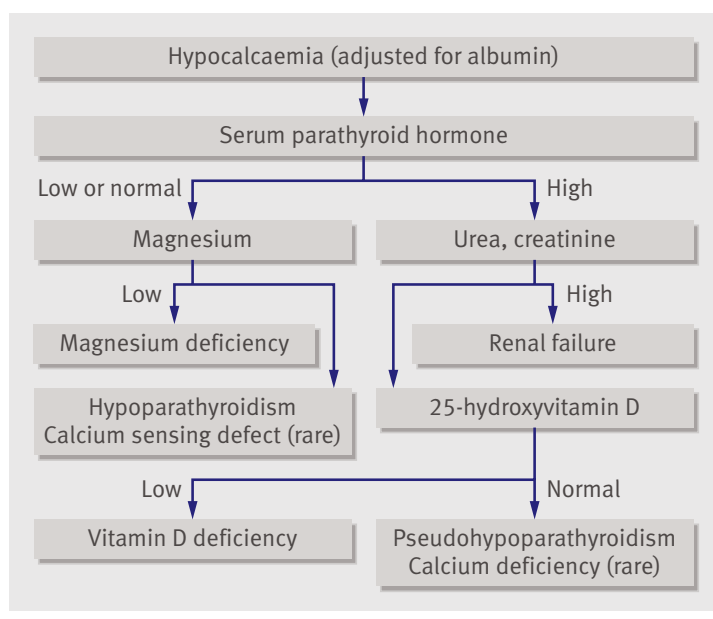

Fig $5 \mid$ Algorithm for requesting investigations to elucidate the cause of hypocalcaemia

feedback of calcium or low if these glands are the cause of the problem. "Normal" concentrations of parathyroid hormone in this context are inappropriate and thus abnormal. A high concentration of parathyroid hormone in the presence of normal renal function suggests deficiency of vitamin D or calcium malabsorption. A low concentration usually indicates hypoparathyroidism. Parathyroid hormone concentrations can be normal in hypoparathyroidism, but they are usually within the lower part of the reference range.

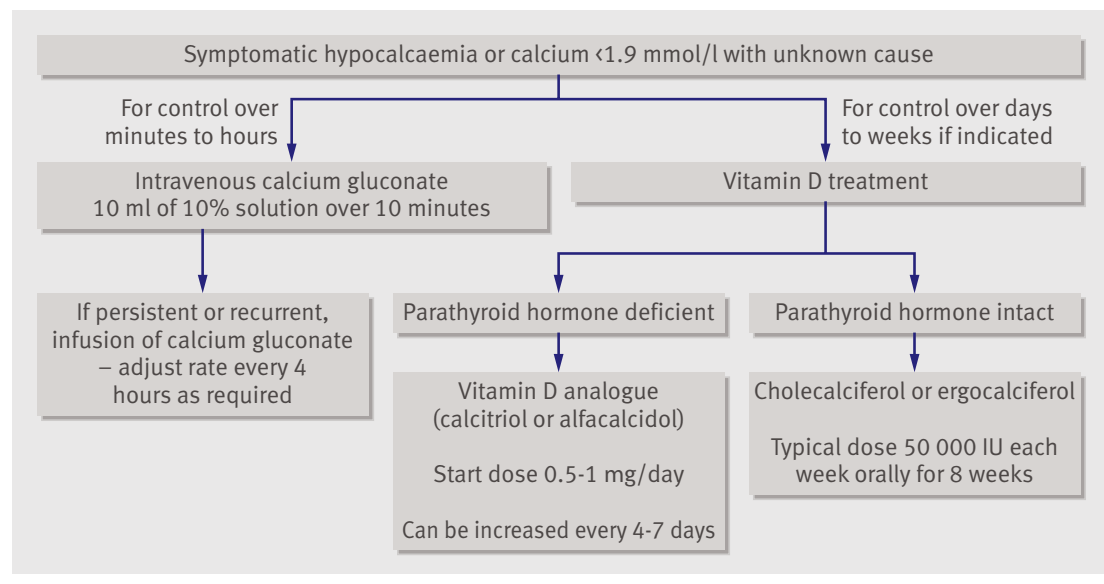

Fig 6 | Algorithm for managing acute hypocalcaemia. In an emergency setting parenteral calcium is likely to be needed. If medium or long term treatment is needed, vitamin D or one of its analogues should be given ${ }^{18}$

\section{ADDITIONAL EDUCATIONAL RESOURCES}

Endotext online textbook (www.endotext.org/parathyroid/)—Website that covers a range of bone and mineral disorders

Osteoporosis and bone physiology. Osteomalacia (http://courses.washington.edu/ bonephys/hypercalU/opmal2.html)—Educational website with resources relating to osteomalacia

Arthritis Research Campaign. Osteomalacia resources (www.arc.org.uk/arthinfo/)Educational material on osteomalacia, including information sheets in a range of languages
Parathyroid hormone concentrations can also be inappropriately normal in hypomagnesaemia (see below) or when the extracellular calcium sensing receptor has increased sensitivity (an autosomal dominant genetic disorder). ${ }^{17}$

A raised serum alkaline phosphatase suggests osteomalacia as a result of vitamin $\mathrm{D}$ deficiency. The possibility of metastatic cancer, with sclerotic metastases causing rapid absorption of calcium into the skeleton, should also be excluded. Parathyroid hormone stimulates renal phosphate clearance, so serum phosphate should be low in non-parathyroid disease but high in parathyroid hormone deficiency. Measuring phosphate has limited value because it varies diurnally and is affected by food intake. Renal function should be measured because the kidney is central to several aspects of calcium homoeostasis.

Other tests may be needed in some situations. Serum 25-hydroxyvitamin D concentrations are useful in confirming vitamin $\mathrm{D}$ deficiency when it presents atypically, and it should be assessed in patients with possible pseudohypoparathyroidism. Serum magnesium is important for the synthesis and release of parathyroid hormone. ${ }^{18}$ In hypomagnesaemia, the release of parathyroid hormone is inhibited, leading to (potentially severe) hypocalcaemia. The prevalence of hypomagnesaemia in a large primary care cohort was $2 \%,{ }^{19}$ but in intensive care it can be up to $65 \%{ }^{20}$ Poor nutrition associated with chronic alcohol use, prolonged diarrhoea, and treatment with diuretics and certain chemotherapeutics (such as cisplatin) causes hypomagnesaemia. Recognition of hypomagnesaemia is important because in this setting it is difficult to reverse hypocalcaemia without magnesium repletion.

\section{How is hypocalcaemia treated?}

The optimal management of hypocalcaemia has not been examined in clinical trials. A core of accepted practice exists, however. The approach to treatment depends on speed of onset, biochemical severity, and clinical features.

\section{Acutely presenting hypocalcaemia}

Neuromuscular irritability with hypocalcaemia requires prompt management in hospital and treatment with intravenous calcium. Asymptomatic patients with corrected serum calcium less than $1.9 \mathrm{mmol} / \mathrm{l}$ may develop serious complications and admission should be considered. Figure 6 shows an algorithm for managing acute hypocalcaemia in adults that is based on clinical experience and expert recommendations. ${ }^{21}$ Calcium gluconate is the preferred form of intravenous calcium because calcium chloride is more likely to cause local irritation. One or two $10 \mathrm{ml}$ ampoules of $10 \%$ calcium gluconate should be diluted in $50-100 \mathrm{ml}$ of $5 \%$ dextrose and infused slowly over 10 minutes. Electrocardiographic monitoring is recommended because dysrhythmias can occur if correction is too rapid. Treatment can be repeated until symptoms have cleared. Often this offers only temporary relief, and continuous 


\section{SUMMARY POINTS}

Serum calcium concentrations must be interpreted in the context of serum albumin concentrations

Patients with a corrected calcium $<1.9 \mathrm{mmol} / \mathrm{l}$ should be urgently assessed for admission, investigation, and treatment

Strong risk factors for vitamin D deficiency include pigmented skin, skin coverage, or both

In patients with low magnesium, hypocalcaemia is unlikely to be corrected without first correcting the low magnesium

Patients with parathyroid hormone deficiency need calcitriol or alfacalcidol rather than conventional doses of colecalciferol or ergocalciferol

\section{UNANSWERED QUESTIONS}

What is the optimal long term treatment of hypoparathyroidismactive treatment with vitamin D or replacement of parathyroid hormone?

What is the best way to prevent vitamin $\mathrm{D}$ deficiency-dietary supplements, food fortification, or adequate sun exposure?

Do low concentrations of vitamin D predispose people to malignancy or inflammatory disease, as epidemiological studies suggest? administration of a dilute solution of calcium may be needed to prevent recurrence of hypocalcaemia. Ten $10 \mathrm{ml}$ ampoules of $10 \%$ calcium gluconate in 1 litre of $5 \%$ dextrose or $0.9 \%$ saline may be given at an initial infusion rate of $50 \mathrm{ml} /$ hour, with the aim of maintaining serum calcium at the lower end of the reference range. ${ }^{21} \mathrm{An}$ infusion of $10 \mathrm{ml} / \mathrm{kg}$ of this solution over four to six hours will increase serum calcium by $0.3-0.5 \mathrm{mmol} / \mathrm{l}$. Oral calcium supplements should be given concurrently, and -if parathyroid hormone is deficient or non-functional — calcitriol should be given (for example, $1 \mu \mathrm{g} /$ day).

Patients taking digoxin have increased cardiac sensitivity to fluctuations in serum calcium, so intravenous calcium administration should be more cautious in this setting, with careful electrocardiographic monitoring. Patients with hypocalcaemia related to hypomagnesaemia will need magnesium replacement.

\section{Persistent hypocalcaemia}

With milder degrees of hypocalcaemia, treatment depends on the underlying cause. In vitamin D deficiency, treatment should be with vitamin $\mathrm{D}$, either ergocalciferol (vitamin D2) or cholecalciferol (vitamin D3; these two forms of vitamin D can be considered identical in this setting). Most available vitamin D preparations include calcium, and two tablets of calcium with vitamin D, each containing 400 IU vitamin $\mathrm{D}$, are usually given daily. This dose of vitamin $\mathrm{D}$ is relatively low, and patients with symptomatic vitamin $\mathrm{D}$ deficiency or those who fail to respond can be treated in the short term with higher doses, such as 50000 IU orally once a week for eight weeks or 300000 IU intramuscularly every three months. ${ }^{22}$

In patients with hypoparathyroidism, the above doses of vitamin $\mathrm{D}$ are ineffective because parathyroid hormone is needed for conversion to 1,25-dihydroxyvitamin D. Thus calcitriol or alfacalcidol is needed. Typical starting doses are $0.5 \mu \mathrm{g}$ of calcitriol or $1 \mu \mathrm{g}$ of alfacalcidol each day, with doses being increased every four to seven days to achieve a serum calcium in the lower part of the reference range. Once a stable calcium concentration is achieved, concentrations should be monitored every three to six months. The main long term risk is the development of nephrocalcinosis as a result of hypercalciuria. Urinary calcium excretion should be monitored annually and if high the dose of vitamin $\mathrm{D}$ may need to be reduced.

In hypocalcaemia caused by malabsorption, the underlying problem should be treated if possible. Patients with coeliac disease should receive calcium and vitamin D orally and should comply with a gluten free diet. ${ }^{23}$

\section{Conclusion}

Patients with hypocalcaemia need careful and timely assessment and intervention to ensure that the risk of life threatening complications is minimised. Where doubt exists and in symptomatic patients, urgent referral to secondary care for further investigation and treatment is required.

Contributors: NJLG and MSC both searched the literature. NJLG wrote the initial draft, which was further developed by both authors. The final version was approved by both authors. NJLG is guarantor.

Competing interests: None declared.

Provenance and peer review: Commissioned; externally peer reviewed.

1 Aishah AB, Foo YN. A retrospective study of serum calcium levels in a hospital population in Malaysia. Med J Malaysia 1995;50:246-9.

2 Hastbacka J, Pettila V. Prevalence and predictive value of ionized hypocalcemia among critically ill patients. Acta Anaesthesiol Scand 2003;47:1264-9.

3 Holick MF. Vitamin D deficiency. N Engl J Med 2007;357:266-81.

4 Mughal MZ, Salama H, Greenaway T, Laing I, Mawer EB. Lesson of the week: florid rickets associated with prolonged breast feeding without vitamin D supplementation. BM/ 1999:318:39-40.

5 Hahn TJ, Hendin BA, Scharp CR, Haddad JG Jr. Effect of chronic anticonvulsant therapy on serum 25-hydroxycalciferol levels in adults. N Engl / Med 1972;287:900-4.

6 Tolman KG, Jubiz W, Sannella J,, Madsen JA, Belsey RE, Goldsmith RS et al. Osteomalacia associated with anticonvulsant drug therapy in mentally retarded children. Pediatrics 1975;56:45-50.

7 Marx SJ. Hyperparathyroid and hypoparathyroid disorders. N Engl J Med 2000;343:1863-75.

8 Bastepe M, Juppner H. Pseudohypoparathyroidism and mechanisms of resistance toward multiple hormones: molecular evidence to clinical presentation. J Clin Endocrinol Metab 2003;88:4055-8.

9 Peter R, Mishra V, Fraser WD. Severe hypocalcaemia after being given intravenous bisphosphonate. BMJ 2004;328:335-6.

10 Urbano FL. Signs of hypocalcemia: Chvostek's and Trousseau's. Hosp Physician 2000;36:43-5.

11 Springhouse, ed. Professional guide to signs and symptoms. 5th ed. Philadelphia: Lippincott Williams \& Wilkins, 2006

12 Netter FH. Clinical manifestations of acute hypocalcemia. In: The Ciba collection of medical illustrations. Vol 4. Summit, NJ: Ciba Pharmaceutical Company, 1965:185.

13 Velasco PJ, Manshadi M, Breen K, Lippmann S. Psychiatric aspects of parathyroid disease. Psychosomatics 1999;40:486-90.

14 Ayuk J, Matthews T, Tayebjee M, Gittoes NJ. A blind panic. Lancet 2001;357:1262.

15 Bushinsky DA, Monk RD. Electrolyte quintet: calcium. Lancet 1998;352:306-11.

16 Slomp J, van der Voort PH, Gerritsen RT, Berk JA, Bakker AJ. Albuminadjusted calcium is not suitable for diagnosis of hyper- and hypocalcemia in the critically ill. Crit Care Med 2003;31:1389-93.

17 Lienhardt A, Bai M, Lagarde JP, Rigaud M, Zhang Z, Jiang Y, et al. Activating mutations of the calcium-sensing receptor: management of hypocalcemia. J Clin Endocrinol Metab 2001;86:5313-23.

18 Anast CS, Winnacker JL, Forte LR, Burns TW. Impaired release of parathyroid hormone in magnesium deficiency. J Clin Endocrinol Metab 1976;42:707-17.

19 Schimatschek HF, Rempis R. Prevalence of hypomagnesemia in an unselected German population of 16000 individuals. Magnes Res 2001;14:283-90.

20 Ryzen E, Wagers PW, Singer FR, Rude RK. Magnesium deficiency in medical ICU population. Crit Care Med 1985;13:19-21.

21 Thakker RV. Parathyroid disorders and diseases altering calcium metabolism. In: Warrall D, Cox T, Firth J, Benz E, eds. Oxford textbook of medicine. 4th ed. Oxford: Oxford University Press, 2003.

22 Malabanan A, Veronikis IE, Holick MF. Redefining vitamin D insufficiency. Lancet 1998;351:805-6.

23 Hopper AD, Hadjivassiliou M, Butt S, Sanders DS. Adult coeliac disease. $B M /$ 2007;335:558-62. 